\title{
ICORG
}


Country: Ireland

\section{Group: The All-Ireland Clinical Oncology Research Group (ICORG)}

Chair:

Dr J. Kennedy

Department of Haematology/Oncology, Hospital 1

St James's Hospital, James St.

DUBLIN 8

EIRE

Tel: +35314162169

Fax: + 35314530557

Email: jkennedy@stjames.ie

Vice-Chair: $\quad$ Dr P. Browne

Department of Haematology

St James's Hospital, James St.

DUBLIN 8

EIRE

Tel: +35314162167

Fax: +35314103428

Email: PBrowne@stjames.ie

Co-Chairs: $\quad$ Professor J. Crown

Chairman, Breast Disease Specific Subgroup

Department of Medical Oncology

St Vincent's University Hospital

Elm Park, DUBLIN 4

EIRE

Tel: +35312094839

Fax: +35312837719

Email: john.crown@icorg.ie

Dr J. O'Sullivan

Radiation Modality Chair

Belfast City Hospital

North Ireland Cancer Clinical Trials Unit

East Podium, C-Floor

Lisburn Road

BELFAST BT9 7AB

UNITED KINGDOM

Professor A. Hill

Surgical Modality Chair

Beaumont Hospital

Beaumont Road

DUBLIN 9

EIRE

Email: arnoldhill@beaumont.ie 
Dr M. Eatock

Medical Modality Chair

Department of Oncology

Belfast City Hospital

51 Lisburn Road

BELFAST BT9 7AB

UNITED KINGDOM

Tel: +442890329241

Fax: +44 2890263514

Email: martin.eatock@bch.n-i.nhs.uk

Dr E. Vandenberghe

Haematological Modality Leader

St James's Hospital, James St.

DUBLIN 8

EIRE

Tel: +35314104545

Fax: +35314103428

Email: evandenberghe@stjames.ie

Professor M. Lawlor

Research professional Modality Leader

St James's Hospital, James St.

DUBLIN 8

EIRE

Tel: + 35314103588

Fax: +35314103476

Email: mlawler@stjames.ie

ICORG Central

\section{Office:}

B. Moulton

Chief Executive Officer

120 Pembroke Road

DUBLIN 4

EIRE

Tel: +35316677211

Fax: +35316697869

Email: brian.moulton@icorg.ie

T. O'Shea

Project Manager - Breast

120 Pembroke Road

DUBLIN 4

EIRE

Email: Tanya.oshea@icorg.ie

Website: www.icorg.ie 